\title{
Super-LOTIS Early Time Optical Counterpart Measurements
}

H. S. Park, R. A. Porrata, G. G. Williams, E. Ables, D. L. Band, S. D. Barthelmy, R. M. Bionta, T. L. Cline, D. H. Ferguson, G. J. Fishman, N. Gehrels, D. Hartman, K. Hurley, C. Kouveliotou, C. A. Meegan, R. Nemiroff, W. Pereira

This article was submitted to

The Fifth Huntsville Gamma Ray Burst Symposium, Huntsville, Alabama, October 18 - 22, 1999

U.S. Department of Energy

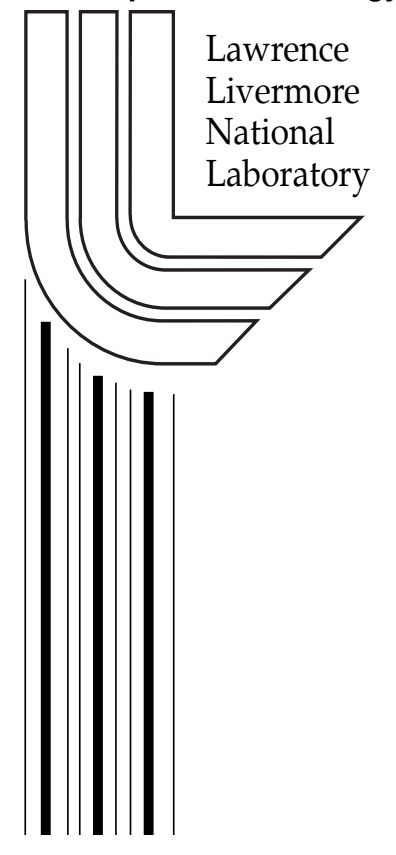




\section{DISCLAIMER}

This document was prepared as an account of work sponsored by an agency of the United States Government. Neither the United States Government nor the University of California nor any of their employees, makes any warranty, express or implied, or assumes any legal liability or responsibility for the accuracy, completeness, or usefulness of any information, apparatus, product, or process disclosed, or represents that its use would not infringe privately owned rights. Reference herein to any specific commercial product, process, or service by trade name, trademark, manufacturer, or otherwise, does not necessarily constitute or imply its endorsement, recommendation, or favoring by the United States Government or the University of California. The views and opinions of authors expressed herein do not necessarily state or reflect those of the United States Government or the University of California, and shall not be used for advertising or product endorsement purposes.

This is a preprint of a paper intended for publication in a journal or proceedings. Since changes may be made before publication, this preprint is made available with the understanding that it will not be cited or reproduced without the permission of the author.

This report has been reproduced directly from the best available copy.

Available electronically at http://www.doc.gov/bridge

Available for a processing fee to U.S. Department of Energy

And its contractors in paper from

U.S. Department of Energy

Office of Scientific and Technical Information

P.O. Box 62

Oak Ridge, TN 37831-0062

Telephone: (865) 576-8401

Facsimile: (865) 576-5728

E-mail: reports@adonis.osti.gov

Available for the sale to the public from

U.S. Department of Commerce

National Technical Information Service

5285 Port Royal Road

Springfield, VA 22161

Telephone: (800) 553-6847

Facsimile: (703) 605-6900

E-mail: orders@ntis.fedworld.gov

Online ordering: http://www.ntis.gov/ordering.htm

\section{OR}

Lawrence Livermore National Laboratory

Technical Information Department's Digital Library

http:/ / www.llnl.gov/tid/Library.html 


\title{
Super-LOTIS \\ Early Time Optical Counterpart Measurements
}

\author{
H. S. Park ${ }^{1}$, R. A. Porrata ${ }^{1}$, G. G. Williams ${ }^{2}$, E. Ables ${ }^{1}$, D. L. \\ Band $^{5}$, S. D. Barthelmy ${ }^{3}$, R. M. Bionta ${ }^{1}$, T. L. Cline $e^{3}$, D. H.
} Ferguson $^{6}$, G. J. Fishman ${ }^{4}$, N. Gehrels ${ }^{3}$, D. Hartmann ${ }^{2}$, K. Hurley ${ }^{7}$, C. Kouveliotou ${ }^{4}$, C. A. Meegan ${ }^{4}$, R. Nemiroff ${ }^{8}$, W. Pereira ${ }^{8}$

${ }^{1}$ Lawrence Livermore National Laboratory, Livermore, CA 94550

${ }^{2}$ Dept. of Physics and Astronomy, Clemson University, Clemson, SC 29634-1911

${ }^{3}$ NASA/Goddard Space Flight Center, Greenbelt, MD 20771

${ }^{4}$ NASA/Marshall Space Flight Center, Huntsville, AL 35812

${ }^{5}$ Los Alamos National Laboratory, Los Alamos, NM 87545

${ }^{6}$ Dept. of Physics, California State University at Hayward, Hayward, CA 94542

${ }^{7}$ Space Sciences Laboratory, University of California, Berkeley, CA 94720-7450

${ }^{8}$ Dept. of Physics, Michgan Technological University, Houghton, MI 49931

\begin{abstract}
We present an update on our ongoing effort to establish a dedicated observation program with an automated 0.6 meter telescope system that can detect GRB optical signals from $30 \mathrm{~s}$ to many hours after the start of the burst. The Super-LOTIS telescope has a $0.8 \times 0.8^{\circ}$ field-of-view, is sensitive to V $17 \sim 19$ objects, depending on the integration times, and will be placed at the Kitt Peak National Observatory. This paper presents technical aspects of this telescope and first results from initial operations at LLNL. Utilizing real-time coordinates from BATSE, BeppoSAX, XTE, IPN, HETE-2 and INTEGRAL, our LOTIS and SLOTIS systems will measure prompt GRB optical light curves that will enhance our understanding of GRBs.
\end{abstract}

\section{INTRODUCTION}

Nearly thirty years after the discovery of GRBs, x-ray, optical and radio afterglows now have been observed for a dozen of bursts during the last two years [1], [2], [3], [4]. These observations finally determined that GRBs are at cosmological distances and have established some of the GRB parameters such as energy, ambient environment and dynamics. However, there is still very little understanding of the nature of the GRB progenitors. Recent detection of a prompt optical signal [5] is inconsistent with the brightness and spectrum of the later time afterglows [6]. This amplifies our needs to measure more simultaneous optical counterparts 
associated with GRBs. Prompt optical activity measurements will provide clues to understanding of GRB production mechanism [7].

To search for simultaneous optical counterparts of GRBs, we are operating an automated wide field-of-view telescope at Lawrence Livermore National Laboratory (LLNL) to rapidly image GRB coordinate error boxes distribured by the Gammaray burst Coordinate Distribution Network (GCN) [8]. The LOTIS results are given in many papers including the one presented at this conference [9], [10], [11], [12]. In this paper, we describe our next generation prompt optical measurment experiment, Super-LOTIS, that will search for quasi-simultaneous GRB optical signals starting $30 \mathrm{~s}$ to many hours after the burst with a sensitivity of $\mathrm{V} 17 \sim 19$.

\section{SUPER-LOTIS}

The telescope is a Boller and Chivens 0.6 meter reflective telescope of $\mathrm{f} / 3.5$. Figure 1 shows the telescope. In order to make it dedicated and automated GRB follow-up telescope, we added computer controllable drives. These drives can point to any part of the sky within $30 \mathrm{~s}$ upon receipt of a GCN trigger. We also designed and fabricated a custom 4-element coma corrector to match the point spread function to the pixel scale at the corners of the imaging CCD. The sensor is a LOTIS CCD camera utilizing a Loral 442A 2048 x 2048 CCD (15 x $15 \mu \mathrm{m}$ pixels) with LLNL built readout electronics. The CCD has thermo electric cooling (to $-30^{\circ} \mathrm{C}$ ) to minimize dark current and readout noise. Super-LOTIS has a $0.84 \times 0.84^{\circ}$ field-

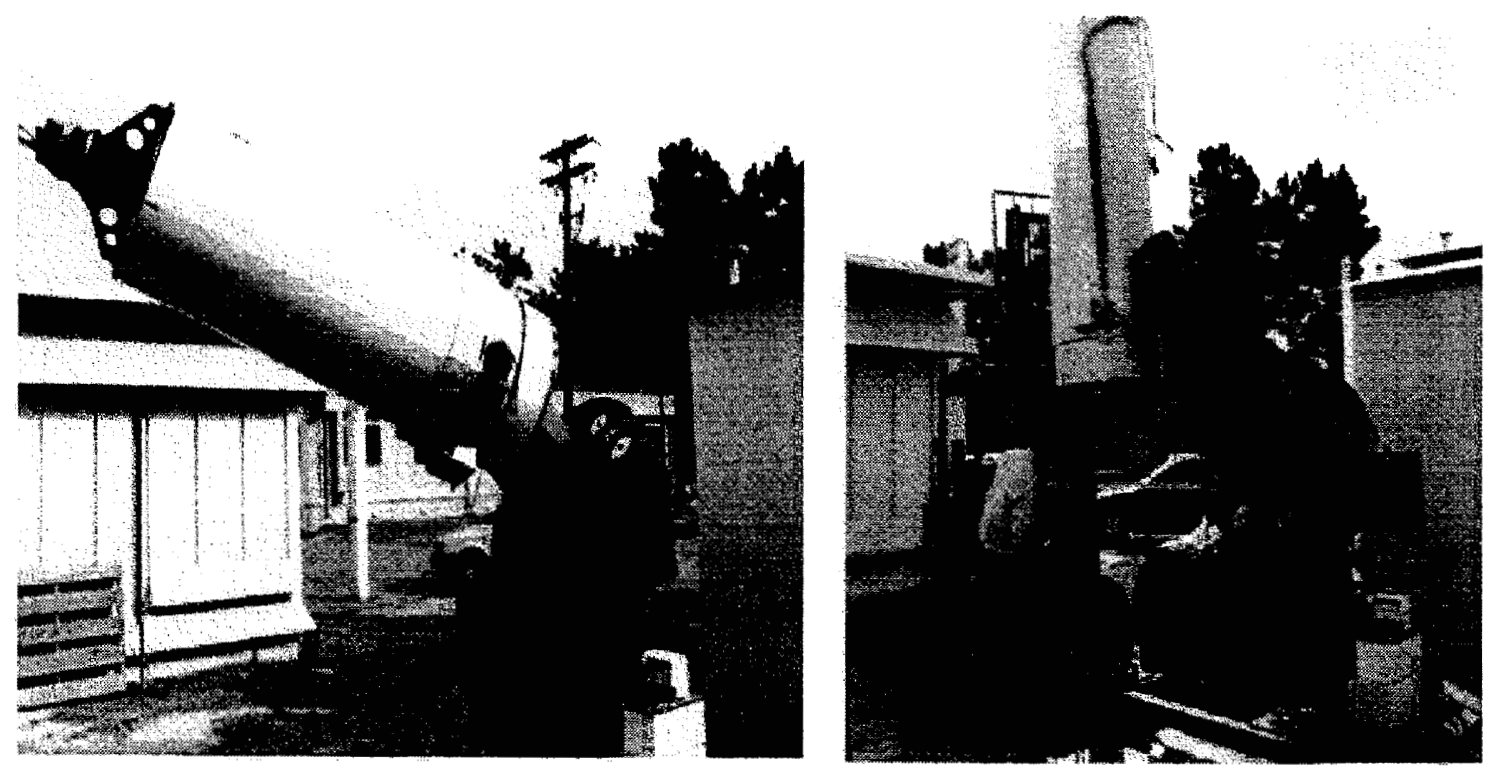

FIGURE 1. Super-LOTIS Boller and Chivens 0.6 meter reflective telescope. We converted it into a dedicated prompt GRB counterpart search telescope by adding computer controlled motors, a. $2 \mathrm{~K} \times 2 \mathrm{~K} \mathrm{CCD}$ camera and an automated data acquisition system. 
of-view ( 1.5 arcsec/pixel) sufficient for most GRB satellite triggers distributed by the GCN.

Our data acqusition system includes custom readout electronics, a custom hardware power control unit, a weather station and a housing control unit. An extensive on-line scheduling software has been written to handle various triggers. Priority is given to the most recent trigger that has smallest error box. For example, in response to a GCN "Original" trigger, which has only a $5 \mathrm{~s}$ delay but a large $15^{\circ}$ error box, the telescope begins to systematically acquire a mosaic of images covering the error box. When refined positions are received, i.e. LOCBURST, XTE, BeppoSAX, or HETE 2 triggers, the telescope moves to that region and stays at that location the rest of the night. Our scanning strategy and automation allows us to record GRB optical activity as early as $30 \mathrm{~s}$.

\section{FIRST LIGHT AND FIRST EVENT OF SUPER-LOTIS}

We have completed installing the motor drivers, coma corrector, CCD camera and the data acquisition system. We imaged the night sky successfully the first time on Feb. 25, 1999.

While we were testing the on-line software Super-LOTIS obtained early time

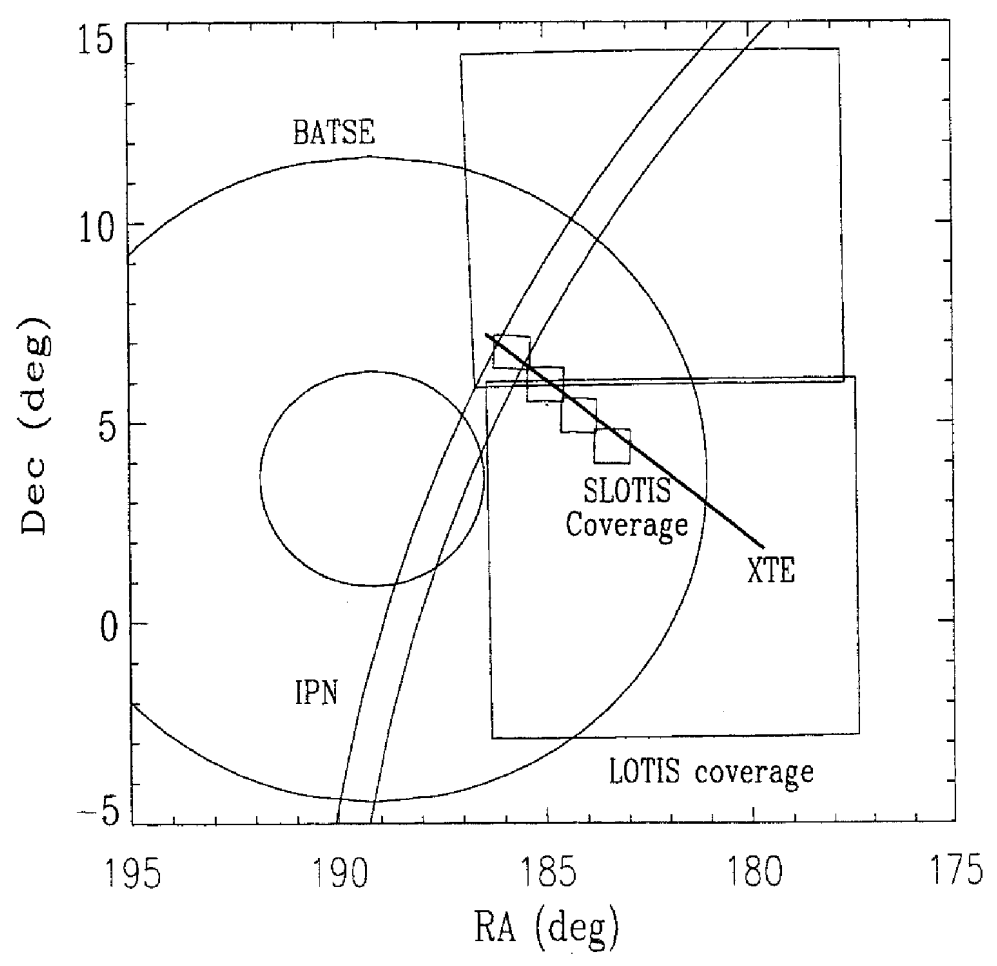

FIGURE 2. Super-LOTIS coverage of GRB990308. 
observations of the error box of GRB 990308 (BATSE Trig. 7457). This event was detected and localized by the XTE/All-Sky Monitor. Super-LOTIS began a raster scan around the LOCBURST GCN coordinates $1700 \mathrm{~s}$ after the start of the burst. Four Super-LOTIS images (30 s integration; t=1694 s, 1809 s, 2620 s, 3923 s) covered most of the XTE/ASM error box within the BATSE 2- $\sigma$ error circle.

The coverage of this event is shown in Figure 2. Here the circles represent the BATSE 1 and 3- $\sigma$ errors; the arc represent the IPN error, and the thin line represent XTE observation. The large boxes represent LOTIS coverage of this event at 132 $\mathrm{s}$ after the burst; and the small boxes are the Super-LOTIS coverage during the raster scan. We searched for optical transient in the area where the error boxes overlap. Even though an optical afterglow for this event has been reported at 3.28 and $3.47 \mathrm{hr}$ after the burst [13], Super-LOTIS detected no fading or flaring objects brighter thatn $\mathrm{V}>15.3$ at 28.2 minutes. The weather condition and the usage of an uncooled prototype CCD camera at the time prevented us from reaching deeper limits.

We have shown that the Super-LOTIS is already operating and capable of responding to GRB triggers. The Super-LOTIS telescope is currently at LLNL for integration undergoing final integration. We will place it at Kitt Peak National Observatory in early 2000 and will be ready for the HETE 2 Coodinate Distribution. With the LOTIS and Super-LOTIS systems, we will be able to cover GRB optical activity from $10 \mathrm{~s}$ to many hours to a magnitude level of V $14 \sim 19$. With HETE 2 and other prompt GRB coordinate distributing satellites, we will be able to measure early time optical activity. Once we detect an optical transient, we will also be able to promptly alert other telescopes.

This work was performed under the auspices of the U.S. Department of Energy by the University of California, Lawrence Livermore National Laboratory under Contract No. W-7405-Eng-48.

\section{REFERENCES}

1. Costa, E., et al., Nature, 387, 783 (1997).

2. Heise, J., et al., IAU Circ., 6654 (1997).

3. van Paradijs, J., et al., Nature, 386, 686 (1997).

4. Frail, D., et al., Nature, 389, 261 (1997).

5. Akerlof, C., et al., Nature, 398, 400 (1999).

6. Briggs, M., et al., ApJ, 524, 82 (1999).

7. Sari, R., et al., ApJ, 517,L109 (1999).

8. Barthelmy, S., et al., AIP Conf. Proc. 428, 99 (1998).

9. Williams, G., et al., These Proceedings (2000).

10. Park, H., et al., ApJ, 490, L21 (1997).

11. Park, H., et al., $A p J, 490,99$ (1997).

12. Williams, G., et al., ApJ, 519, L25 (1997).

13. Schaefer, B., et al., ApJ, 524,L103 (1999). 